\title{
Towards personalized medicine: Photoacoustic imaging of glutathione enables companion diagnosis and targeted treatment of lung cancer
}

\author{
Melissa Y. Lucero and Jefferson Chan* \\ Department of Chemistry and Beckman Institute for Advanced Science and Technology, University of \\ Illinois at Urbana-Champaign, Urbana, IL 61801, United States
}

\begin{abstract}
Companion diagnostics (CDx) represent a new frontier in personalized medicine that promises to improve treatment outcomes by matching therapies to patients. Currently, these tests are limited in scope and cannot report on real-time changes associated with disease progression and remediation. To address this, we have developed the first photoacoustic imaging-based CDx (PACDx) for the selective detection of elevated glutathione (GSH) in lung cancer. Since GSH is abundant in most cells, we utilized a physical organic approach to precisely tune the chemical reactivity to distinguish between normal and pathological states. In blinded studies, PACDx was applied to identify mice bearing lung tumors. Moreover, we designed a matching prodrug, PARx, that utilizes the same mechanism to release a chemotherapeutic with a PA readout. We demonstrate that PARx can inhibit tumor growth without off-target toxicity in a lung cancer xenograft model. We envision that this work will establish a new standard for personalized medicine by employing a unique imaging-based approach.
\end{abstract}

\section{Introduction}

Despite progress in the diagnosis, treatment, and management of cancer, nearly 10 million individuals will die each year, with lung cancer being the leading cause. ${ }^{1} \mathrm{~A}$ new frontier in personalized medicine that aims to improve these statistics is the application of companion diagnostics $(C D x) \cdot{ }^{2} \mathrm{CDx}$ are tests that provide essential information for determining which patients may benefit from a particular treatment by detecting biomarkers that are critical for drug activation. ${ }^{3}$ Current FDA-approved CDx are designed for in vitro testing and thus, cannot account for variations in the tumor microenvironment which can impact drug efficacy. To provide accurate, real-time information, it is crucial to develop new molecular imaging-based CDx, as well as corresponding drugs for aberrant cancer properties. However, most clinically approved imaging agents for cancer target cell surface biomarkers through stoichiometric binding events. In addition to poor signal- 
to-noise, binding-based strategies preclude the detection of small molecule targets (e.g., glutathione $(G S H))$. Activity-based sensing (ABS) represents a powerful alternative that relies on selective detection of chemical reactivity. In contrast to fluorescence-based ABS probes that can only operate in the millimeter depth range due to scattering and light attenuation, the corresponding chemical tools for photoacoustic (PA) imaging can be employed as a CDx for in vivo testing. PA is a noninvasive modality that utilizes nearinfrared (NIR) light to induce the generation of an ultrasound signal. Because ultrasound scatters 1000 times less than light in biological tissues, high resolution images (tens of microns) can be obtained in the centimeter range ${ }^{4}$ Label-free PA imaging has already been employed clinically for the detection of breast cancer, ${ }^{5}$ thyroid cancer, ${ }^{6}$ inflammatory arthritis, ${ }^{7}$ and scleroderma. ${ }^{8}$ Recently, our group and others have expanded the scope of PA imaging through the development of acoustogenic ABS probes..$^{9,10}$ Select examples include imaging agents for the detection of metal ion dysregulation, ${ }^{11-14}$ hypoxia, ${ }^{15-18}$ proteases, ${ }^{19,20}$ and signaling molecules (e.g., nitric oxide ${ }^{21,22}$ and hydrogen sulfide ${ }^{23}$ ). In this study we leverage classic principles in physical organic chemistry to guide the development of the first PA imagingbased $\underline{\mathrm{CDx}}(\mathrm{PACDx})$, as well as a matching Gemcitabine-based prodrug (PARx). Application of PACDx and PARx in an unprecedented blind study allowed us to successfully identify and treat mice with lung cancer, respectively.

\section{Results}

\section{Physical organic approach to guide design of PACDx}

GSH is an abundant biological thiol that is essential for maintaining redox homeostasis and detoxifying xenobiotics. ${ }^{24}$ Aberrant changes in the cellular levels of GSH are correlated with a variety of pathologies such as cancer. ${ }^{25}$ Among the various cancer types, non-small cell lung carcinoma has the largest change in GSH levels for both patient derived tissue samples (up to 4-fold) ${ }^{26}$ and human cell lines (up to 7-fold), ${ }^{27,28}$ relative to healthy tissue and non-cancerous cell lines, respectively. In this study we chose to develop a CDx assay for lung cancer based on the detection of GSH via PA imaging.

The design of PACDx features two key elements, a PA dye capable of generating a strong PA signal upon irradiation and a trigger for detecting GSH (Figure 1a). In addition to its large extinction coefficient $(\varepsilon=$ $>10^{4} \mathrm{M}^{-1} \mathrm{~cm}^{-1}$ ) in the near-infrared region, the HD platform was selected owing to its lipophilic cationic 
character which can enhance uptake by tumor cells and render the resultant probe a poor substrate for glutathione S-transferase (GST), an abundant cytosolic enzyme that catalyzes the conjugation of GSH to electrophilic centers. ${ }^{29,30}$ We deemed this to be important since the expression of GST may vary in cancer and therefore, cross-reactivity can confound imaging results. We employed a physical organic approach to develop a precision-tuned, GSH-responsive trigger that can distinguish GSH levels between lesions and healthy tissue. This represents a significant challenge because existing triggers based on disulfide exchange ${ }^{31}$ and $\mathrm{S}_{\mathrm{NAr}}{ }^{32}$ chemistry are too reactive. For instance, installation of the common 2,4dinitrobenzenesulfonate trigger onto our PA platform afforded a probe that could not distinguish GSH levels across several mammalian cell lines (Figure S1) since it was fully activated by only $100 \mu \mathrm{M}$ GSH within several minutes ( 10-fold less than physiological levels) (Figure 1f). To establish a structure-reactivity relationship, we synthesized a panel of nine probes featuring a range of electron withdrawing and donating groups at the para-position. The resultant Hammett plot revealed a linear correlation between $\log \left(\mathrm{k}_{\mathrm{x}} / \mathrm{k}_{\mathrm{H}}\right)$ and the corresponding $\sigma^{-}$constants (Figure 1b). ${ }^{33} \mathrm{~A}$ positive $\rho$ value of 1.10 indicates that electron deficient substituents at this position were more efficient at stabilizing the Meisenheimer complex, resulting in enhanced reactivity. Unfortunately, the most reactive probe in this series ( $p$-nitro) was only partially activated to give a 5.6-fold turn-on response after treatment with $10 \mathrm{mM} \mathrm{GSH}$ for $1 \mathrm{~h}$ (Figure 1g). However, a dose- and time-dependent response was evident. Encouraged by these results, we synthesized an additional four probes with both ortho- and para-electron withdrawing groups to further increase the SNAr reactivity (Figure S2). As anticipated from our structure-reactivity data, installation of a fluoro substituent at the ortho-position resulted in a highly optimized trigger (Figure 1h). Activation of the resulting probe, PACDx, under physiological conditions was completely attenuated. On the other hand, a dose-dependent signal increase up to 31.6-fold was observed when PACDx was incubated with $10 \mathrm{mM}$ GSH for $1 \mathrm{~h}$. Rate constants for the SNAr reaction for PACDx, the p-nitro analog, and the 2,4-dinitro congener were measured at various temperatures to construct the corresponding Eyring plots (Figure 1c). The derived activation parameters for PACDx are $\Delta \mathrm{H}^{\ddagger}=70.5 \mathrm{~kJ} \mathrm{~mol}^{-1}$ and $\Delta \mathrm{S}^{\ddagger}=-117.1 \mathrm{~J} \mathrm{~mol}^{-1} \mathrm{~K}^{-1}$. This data suggests that the SNAr reaction involves a highly ordered transition state, which likely results from effective stabilization of the Meisenheimer complex. The Gibbs free energy of activation $\left(\Delta G^{\ddagger}\right)$ for PACDx was calculated to be $106.8 \mathrm{~kJ} \mathrm{~mol}^{-1}$, which indicates that the SNAr reaction was energetically more favorable than the p-nitro 
analog, but less favorable compared to the 2,4-dinitro congener. Finally, we constructed two Brønsted relationship graphs where the pKa values of the corresponding phenols were plotted against $\Delta \mathrm{G}^{\ddagger}$ (Figure 1d) and $\log (\mathrm{k})$ (Figure 1e). In both cases, we observed a linear correlation with $R^{2}$ values $=1.00$, suggesting that this information can guide probe design involving SNAr chemistry. All pertinent results are summarized in Table S1.

\section{Evaluation of PACDx in vitro and in live cells}

Prior to activation, PACDx does not absorb strongly within the PA window (680 - $950 \mathrm{~nm}$ ); however, treatment with GSH induces a bathochromic shift (Figure 2a). Irradiation at the $\lambda_{\max }(690 \mathrm{~nm})$ yields a strong PA response (Figure 2b). A dose-dependent signal enhancement was observed when PACDx was treated with GSH (Figure 2c and $\mathbf{2 d}$ ). In contrast, there was no notable reactivity when the probe was incubated with cysteine $(200 \mu \mathrm{M})$ or homocysteine $(100 \mu \mathrm{M})$ (Figure S5). Next, we evaluated the performance of PACDx in A549 lung cancer cells, U87 glioblastoma cells and HEK 293 cells (a non-cancerous cell line) using confocal microscopy. PACDx was highly fluorescent in A549 cells with a cytosolic and mitochondrial staining pattern (Figure 2e). However, when cells were pretreated with $N$-ethylmaleimide (NEM), a reagent used to reduce the levels of intracellular thiols, the fluorescence signal was attenuated by $\sim 50 \%$ (Figure 2f). To confirm that the decrease in intensity was due to GSH depletion, a third set of cells were treated with a non-responsive control probe (Ctrl-PACDx, Figure $\mathbf{S 6}$ and Figure $\mathbf{2 g}-\mathbf{h}$ ). A similar trend comparable to A549 cell imaging was observed in U87 cells (Figure S7). When A549 and U87 cells were pretreated with ethacrynic acid, ${ }^{34}$ a potent reversible inhibitor of human GST, no effect on probe activation was observed which suggests activation of PACDx is independent of GST enzymatic activity (Figure S8).

For PACDx to function as an effective CDx, it is essential for it to be able to accurately differentiate the relative GSH levels in lung cancer cells compared to healthy cells. To our delight, the intensity of PACDx in A549 cells was indeed the highest relative to U87 and HEK 293 cells (Figure 2i). However, it is important to note that the signal from the U87 cells was significantly lower than that of the HEK 293 cells. To account for possible variations in probe uptake and retention, we incubated PACDx with cell lysates and obtained consistent results (Figure S9). This suggests that the intracellular levels of GSH is highest in A549 cells 
and lowest in U87 cells. This was confirmed with the established Ellman's assay which allows for accurate quantification of GSH levels in cell lysates (Figure $\mathbf{2 j}$ )..$^{35}$

\section{Design and synthesis of PARx}

Next we turned our attention to designing a prodrug invoking the same GSH-mediated chemistry. We rationalized it would be possible to append a chemotherapeutic to the PACDx core by strategically installing a hydroxymethyl handle ortho to the phenolic alcohol. Upon removal of the trigger, the resultant phenolate intermediate can fragment via a 1,4-elimination pathway to release the drug and the corresponding dye $\left(\mathrm{HD}-\mathrm{CH}_{2} \mathrm{OH}\right.$ ) for PA imaging (Figure 3a). We selected to append Gemcitabine, an FDA-approved drug, because it is commonly used to treat non-small cell lung cancer through the inhibition of DNA synthesis. ${ }^{36,37}$ However, as with many chemotherapeutics, Gemcitabine indiscriminately targets any rapidly dividing cell in the body which results in adverse effects such as severe liver toxicity. ${ }^{38}$ Masking the primary alcohol with a variety of capping groups have led to the development of Gemcitabine-based prodrugs displaying attenuated cytotoxicity until they are activated. ${ }^{39}$ Although we could have directly modified Gemcitabine with our new 2-fluoro-4-nitrobenzenesulfonyl trigger, we wanted to utilize the lipophilic cationic dye for targeting and to leverage the PA imaging capabilities of the resulting prodrug (herein named PARx) to monitor drug release in real-time.

PARx was synthesized starting from the sequential reduction of 2,4-dihydroxybenzaldehyde and TBS protection to afford 16 in $61 \%$ yield. In situ deprotection of the phenolic alcohols with sodium hydride facilitated a nucleophilic substitution and retro-Knoevenagel sequence with $\mathrm{Cy} 7-\mathrm{Cl}$ to obtain 17 . The GSHresponsive trigger was installed, and the primary alcohol was deprotected under acidic conditions to yield 18 (Ctrl-PARx-2) in 46\% over 2-steps. Finally, a chloroformate intermediate was generated using phosgene and then reacted with Gemcitabine to obtain PARx (Supporting information).

\section{Evaluation of PARx in vitro and in live cells}

With PARx in hand, we first confirmed that attaching Gemcitabine did not alter the PA imaging properties or the responsiveness of the GSH trigger (Figure 3b-d, and S10). Interestingly, we observed that PARx consistently yielded a more intense PA signal compared to PACDx, presumably due to the larger PA 
brightness value $\left(\varepsilon \times\left(1-\Phi_{\mathrm{FI}}\right)\right) .{ }^{18}$ We also demonstrated that PARx displayed exceptional selectivity against a panel of metal ions, amino acids, reductants, reactive nitrogen and oxygen species, metabolic liver enzymes, and competing thiols (Figure 3e). Next, we employed MS and NMR analyses to confirm that GSH mediates the release of Gemcitabine (Figures S11 and S12). To further support these results, we prepared a control compound (Ctrl-PARx-1) that is equipped with an unreactive 4-methoxybenzenesulfonyl trigger (Figure S13). MS analysis reveals that both the trigger and carbonate linkage of Ctrl-PARX-1 were stable in the presence of $10 \mathrm{mM} \mathrm{GSH}$ for at least $1 \mathrm{~h}$ (Figure S14). Moreover, PARx could readily distinguish GSH levels in live A549, U87, and HEK293 cells independent of GST activity (Figures 3f, S1516). Lastly, we assessed the cytotoxicity of PARx in A549 cells using the MTT assay and observed dosedependent toxicity that is comparable to free Gemcitabine. On the contrary, treatment with either Ctrl-PARx1 or Ctrl-PARx-2, the cytotoxicity was significantly attenuated (Figure $\mathbf{3 g}$ ). MTT assays in U87 cells established that PARx requires elevated levels of GSH found in A549 cells to effectively mediate Gemcitabine release (Fig S17).

\section{In vivo imaging of GSH and tumor inhibition}

To evaluate the in vivo efficacy of the PACDx and PARx pair, we established a A549 xenograft model of lung cancer. After the tumors had grown to $\sim 100 \mathrm{~mm}^{3}$, we administered PACDx via intratumor injection. Irradiation at $680 \mathrm{~nm}$ (the optimal in vivo wavelength) $1 \mathrm{~h}$ post-injection resulted in a $\sim 1.5$-fold PA turn-on response relative to the control flank. Next, we introduced PARx via systemic administration to determine its biodistribution profile, as well as potential off-target cytotoxic effects. We harvested tissue from vital organs $1 \mathrm{~h}$ post-injection for PA imaging analysis. The PA signals from the heart, kidneys, liver and spleen were not statistically different between the treatment and control groups (Figure $\mathbf{4 a}$ and $\mathbf{4 b}$ ). In contrast, the PA intensity was 1.2-fold higher in the tumor for the PARx-treated animals, demonstrating selective activation and drug release. The results from our PA imaging experiments suggest PARx will not have offtarget toxicity. This was confirmed by performing histological staining which revealed that there was minimal toxicity in all organs examined (Figure 4c). However, PARx was highly toxic in tumor tissue as indicated by the decrease in the number of nuclei and size of the tumor cells. In addition, TUNEL staining revealed that the poor morphology observed was due to apoptosis (Figure $\mathbf{4 d}$ ). 
Over a 21-day period, PARx was intratumorally injected into A549 xenografts every 7 days. PA imaging was performed $1 \mathrm{~h}$ after treatment to monitor Gemcitabine release (Figure S18). Compared to control tumors (Figure $\mathbf{5 a}$ and $\mathbf{5 d}$ ), the PA signal was higher in PARx-treated tumors. This indicates PARx activation (Figure $\mathbf{5 b}$ and $\mathbf{5 d}$ ), leading to complete attenuation of tumor growth (Figure 5e). On the contrary, tumors grew up to $\sim 600 \mathrm{~mm}^{3}$ in size when animals received a vehicle control. To evaluate the systemic compatibility of PARx, we retro-orbitally administered PARx every 7 days over the course of a 21-day period (Figures 5e). The inhibition of tumor growth was nearly identical to the results obtained via intratumoral administration. The body weight of each animal was monitored as a measure of general toxicity. Under no treatment conditions did we observe any loss of weight (Figure 5f) or change in behavior. ${ }^{40}$ To further challenge the selectivity of PARx, we increased the dosing frequency to once every 3 days (Figure S19). We hypothesized that we would not see any adverse effects, especially in the liver where severe damage is common with free Gemcitabine. Gratifyingly, we did not observe a change in the body weight (Figure S20) or liver damage.

\section{Application of PACDx in a blind study}

Finally, we designed a blind study to identify animals with lung cancer based on GSH levels to evaluate the performance of PACDx. First, a group of nude mice were implanted with either A549 or U87 cells. After the animals were tagged and randomized by a second researcher, their identity was concealed until the completion of the study. The purpose of implanting U87 cells, as opposed to no tumors, was to ensure that there would be no biasing of the results based on appearance. Next, we measured the PA intensity change after administration of a vehicle to healthy tissue $(1.06 \pm 0.17, n=9)$ to establish a diagnostic threshold (the mean value $+2 \mathrm{SD}$ ). After the tumors had grown to $\sim 100 \mathrm{~mm}^{3}$, PACDx was administered to perform PA imaging (Figure 6a). We identified one animal within one SD of the mean (1.30) and two animals within 2SD of the mean (1.47 and 1.61) (Figure 6b). These three subjects (out of 8 total) were categorized to 'group 1' which we assigned to be mice bearing A549 tumors. The remaining five animals $(0.64,0.84,1.05$, 1.06, and 1.07) were grouped into 'group 2' (Figure 6b). We hypothesized that prodrug treatment of the first group would result in significant tumor attenuation. In contrast, we predicted that the administration of PARx to the second group would have no therapeutic effect owing to low intratumoral GSH levels. All 
animals received PARx via retro-orbital injection once every 3 days for 21 days. The PA turn-on response of group 1 was 1.56-fold higher than that of group 2, indicating greater GSH-mediated activation of PARx (Figure $\mathbf{6 c}$ and $\mathbf{6 d}$ ). At the completion of the study, no significant increase in tumor size was observed in group 1, whereas the tumor volume of group 2 had increased to over $1000 \mathrm{~mm}^{3}$ (Figure 6e) and the mice had lost significant body weight (Figure 6f). When the identity of the animals was revealed, we were delighted to find that we were accurate in each instance, where 7 of 8 mice were correctly assigned with greater than $95 \%$ confidence.

\section{Discussion}

The identification of disease biomarkers and the subsequent use of this information to guide therapeutic decision-making is a hallmark of personalized medicine. In this study, we utilized a physical organic approach to guide the development of the first PA imaging-based CDx for the detection of GSH in lung cancer. The advantage of employing an imaging-based approach rather than a conventional in vitro testing strategy, is the unique ability to visualize changes that occur during disease progression in realtime. It is noteworthy that GSH is typically not considered to be an ideal cancer biomarker because current sensing strategies are too insensitive to distinguish between normal and pathological levels, especially in vivo. We performed precision-tuning to optimize the $S_{N} A r$ reactivity which enabled us to reliably differentiate GSH in the 0.1 to $10 \mathrm{mM}$ range.

We also developed a highly effective prodrug that utilizes the same GSH-mediated activation mechanism to selectively release Gemcitabine from a PA imaging dye. Although we could have directly installed the new trigger onto Gemcitabine, the ability to perform PA imaging provides us with a powerful handle to monitor drug delivery. Moreover, we did not want to drastically alter cellular uptake or biodistribution since the HD dye scaffold has been shown to target tumors, presumably due to the intrinsic positive charge. We envision further exploiting this robust design to selectively deliver other cancer drugs to tumors. With regards to Gemcitabine, it is an FDA-approved drug and one of the first line treatment options for lung cancer. Unfortunately, it is characterized by rapid metabolism, poor bioavailability and low tumor uptake. As a result, frequent doses must be administered to ensure a therapeutic response that can lead to severe liver damage. Since, PARx is only activated in lung cancer cells we were able to demonstrate through 
different dosing regimens (every 7 days versus every 3 days for 21 days) that PARx did not display any offtarget toxicity.

At the onset of this work, our primary objective was to develop a PA-imaging based CDx that could identify subjects with lung cancer based on elevated GSH levels. An additional goal was to employ the CDx to stratify cancer subjects into groups (those that would respond versus those that would not) for subsequent treatment with a matching treatment. Indeed, we were able to design an unprecedented blind study to evaluate the performance of PACDx in the context of these two aims. Through the application of PACDx, we were able to stratify 8 mice bearing different tumor types into two groups. Subsequent treatment with the prodrug demonstrated that only the group suspected of having lung cancer responded to treatment. When the identity of each animal was revealed, we were pleased to find that we could accurately distinguish between different cancer types based on PA imaging. In conclusion, this study showcases the potential use of PA imaging to detect critical biomarkers for effective personalized therapy. This powerful approach has allowed us to predict, prior to treatment, which group of animals will have the greatest therapeutic efficacy when treated with PARx. On another important note, PA monitoring of drug release may also aid in the drug development process since confirmation of drug delivery in real-time is a major challenge. We envision that this work will establish a new strategy for using physical organic chemistry to guide the co-development of PA imaging-based CDx and safe prodrugs.

\section{Acknowledgements}

This work was supported the National Institutes of Health (R35GM133581). M.Y.L acknowledges the Alfred P. Sloan Foundation for financial support. Major funding for the $500 \mathrm{MHz}$ Bruker CryoProbeTM was provided by the Roy J. Carver Charitable Trust (Muscatine, lowa; Grant No. 15-4521) to the School of Chemical Sciences NMR Lab. The Q-Tof Ultima mass spectrometer was purchased in part with a grant from the National Science Foundation, Division of Biological Infrastructure (DBI-0100085). We also acknowledge the Core Facilities at the Carl R. Woese Institute for Genomic Biology for access to the Zeiss LSM 700 confocal microscope and corresponding software. M.Y.L. thanks Dr. Hailey Knox for initial animal training and assistance with the NanoZoomer. We thank Mr. Thomas Bearrood and Dr. Chelsea Anorma for assistance with initial confocal imaging experiments, Mr. Lucas Akin for aid with mass spectrometry 
experiments, Prof. Sayeepriyadarshini Anakk and Ms. Angela Dean for help with interpreting results from H\&E staining experiments, and Prof. Andrew Bennet for helpful discussions.

\section{Author contributions}

M.Y.L. performed all experiments in this study that include chemical synthesis, in vitro characterization, cellular studies, tumor model studies, in vivo imaging, and sample preparation for ex vivo analysis. J.C. assisted with the blinded animal study. M.Y.L. and J.C. analyzed the data and prepared the manuscript. J.C. conceived the project with intellectual contributions from M.Y.L. 
a

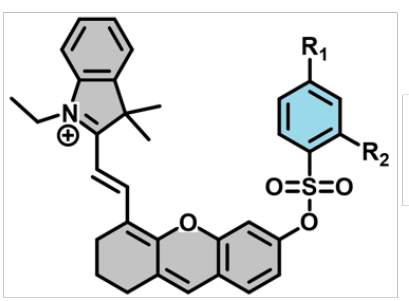

C

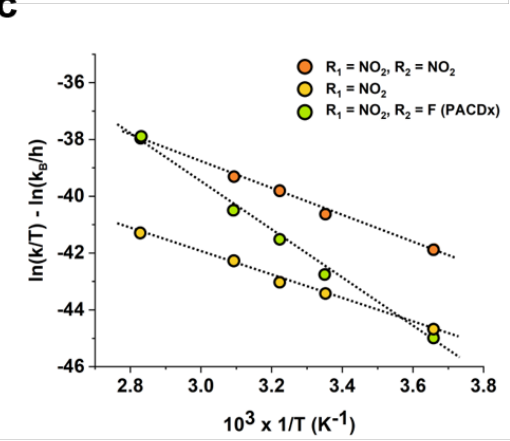

f

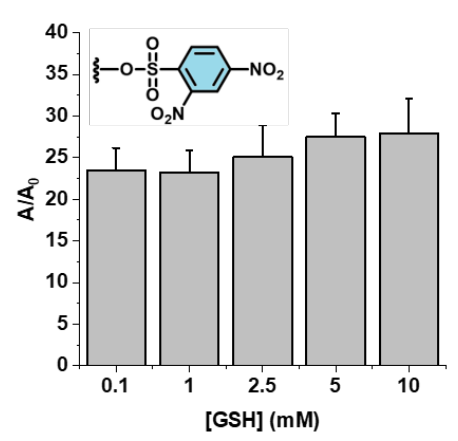

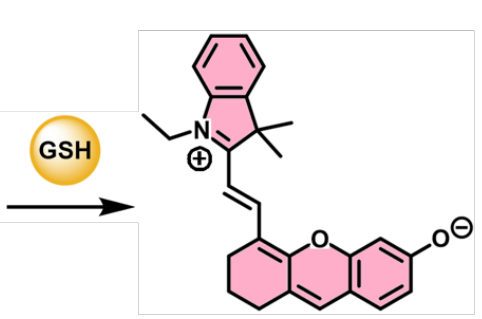

d

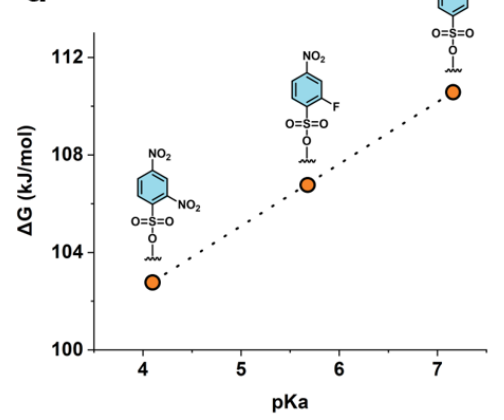

g

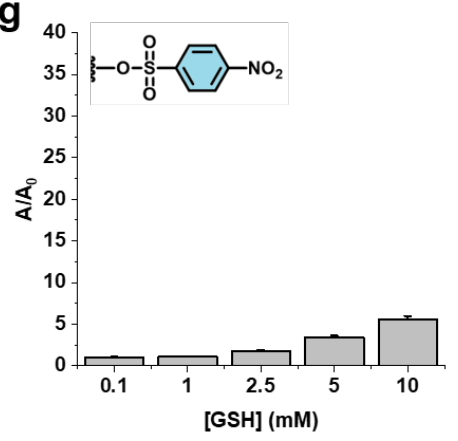

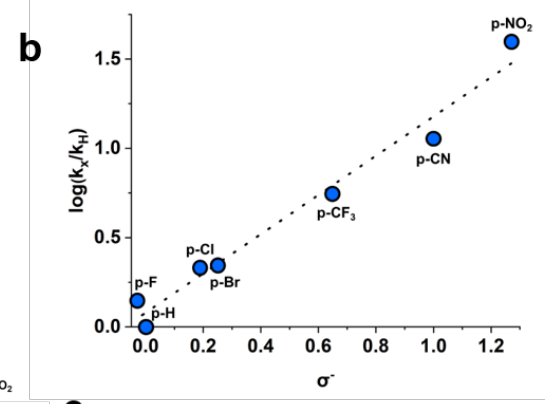

e

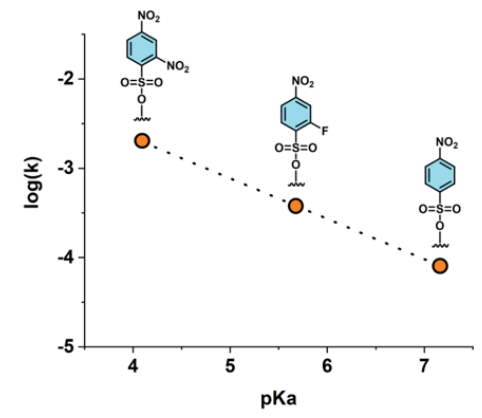

h

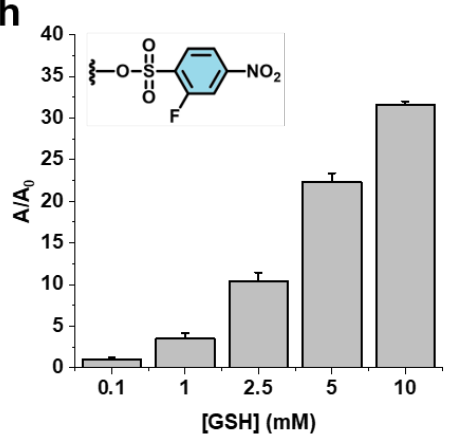

Figure 1. a) General schematic for GSH-responsive photoacoustic companion diagnostics. b) Hammett plot for $\mathrm{S}_{\mathrm{N}} \mathrm{Ar}$ reaction between para-substituted benzenesulfonyls and $10 \mathrm{mM} \mathrm{GSH}$ at $120^{\circ} \mathrm{C}$. Dotted line is the best linear fit of the rate constant data. c) Eyring plots for 2, 5, and PACDx reacting with GSH. Dotted line represents the best linear fit. Brønsted plots indicating the linear relationship between d) $\Delta G$ or e) $\log (k)$ and pKa $\left(R^{2}=1.0\right.$ and 0.99 , respectively) for 2,5 , and $P A C D x$ reacting with $G S H$ at $\left.37^{\circ} \mathrm{C}\right)$. Dose-dependent activation of f) 2, g) 5; and h) PACDx where $[\mathrm{GSH}]=0.1-10 \mathrm{mM}$. All assays performed at $\mathrm{pH} 7.4,70 \%$ $\mathrm{PBS} / \mathrm{MeCN}$. Error bars $=\mathrm{SD}(n=3)$. 

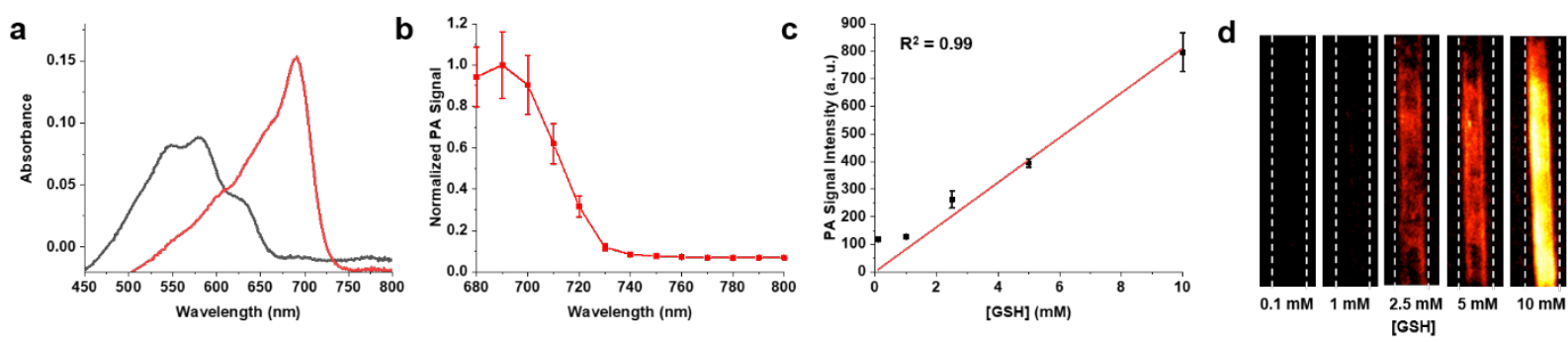

e

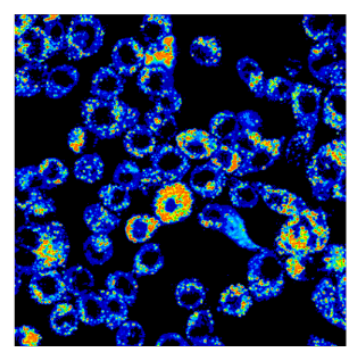

h

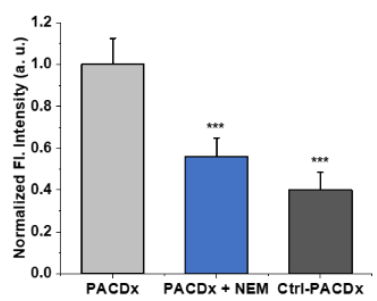

$\mathbf{f}$

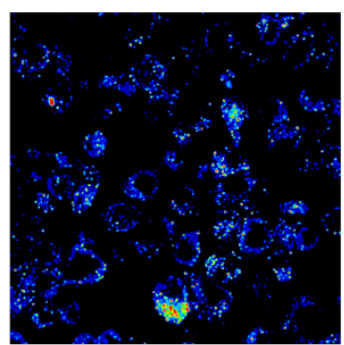

i

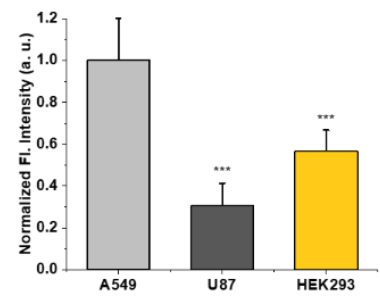

g

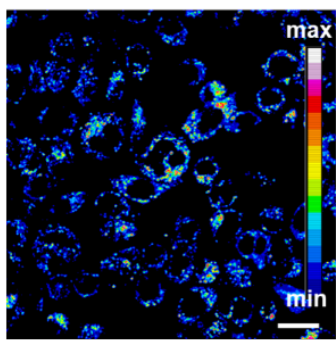

j

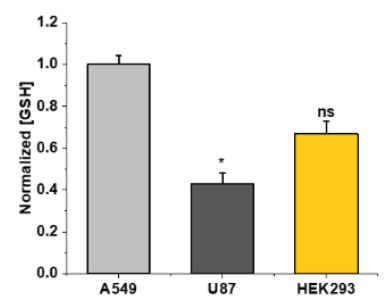

Figure 2. a) Absorbance profile of $5 \mu \mathrm{M}$ PACDx before (black line) and after (red line) treatment with 10 mM GSH. b) PA spectra of $50 \mu \mathrm{M}$ turned over HD dye. c) PA signal $(n=3)$ and d) PA images of PACDx in response to $\mathrm{GSH}$. All in vitro assays were performed at $37{ }^{\circ} \mathrm{C}$ at $\mathrm{pH} 7.4,70 \% \mathrm{PBS} / \mathrm{MeCN}$, data was collected after $1 \mathrm{~h}$. Confocal microscopy image representing e) A549 cells treated with $5 \mu \mathrm{M}$ PACDx for 1 $\mathrm{h}$ at $37^{\circ} \mathrm{C}$, f) A549 cells pre-treated with $1 \mathrm{mM}$ NEM then incubated with $5 \mu \mathrm{M}$ PACDx for $1 \mathrm{~h}$ at $37^{\circ} \mathrm{C}$, and g) A549 cells treated with $5 \mu \mathrm{M}$ Ctrl-PACDx for $1 \mathrm{~h}$ at $37^{\circ} \mathrm{C}$. Scale bar represents $20 \mu \mathrm{m}$. h) Normalized fluorescence intensity obtained from cell imaging under conditions represented in Figure 2e-g. Error bars $=\mathrm{SD}(n \geq 3)$. i) Normalized fluorescence intensity obtained from cell imaging A549, U87, and HEK293 cells with $5 \mu \mathrm{M}$ PACDx for $1 \mathrm{~h}$ at $37^{\circ} \mathrm{C}$. Error bars $=\mathrm{SD}(n \geq 3)$. j) Normalized [GSH] obtained from Ellman's assay. Error bars $=\mathrm{SD}(n=2)$. Statistical analysis was performed using Student's t-test, ${ }^{* * *}: \mathrm{p}<0.001,{ }^{* *}$ : $p<0.01,{ }^{*}: p<0.05$ 
a
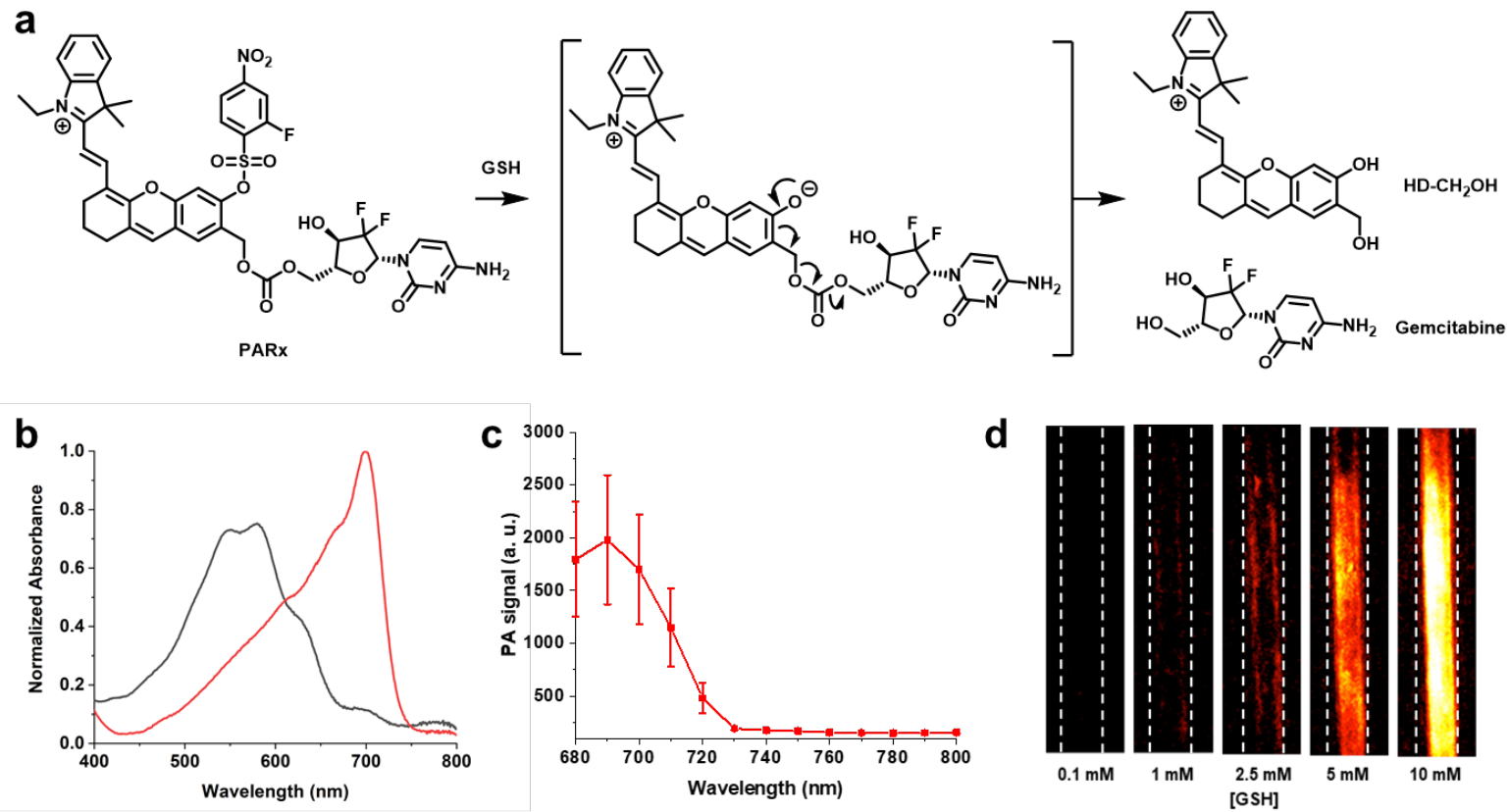

d
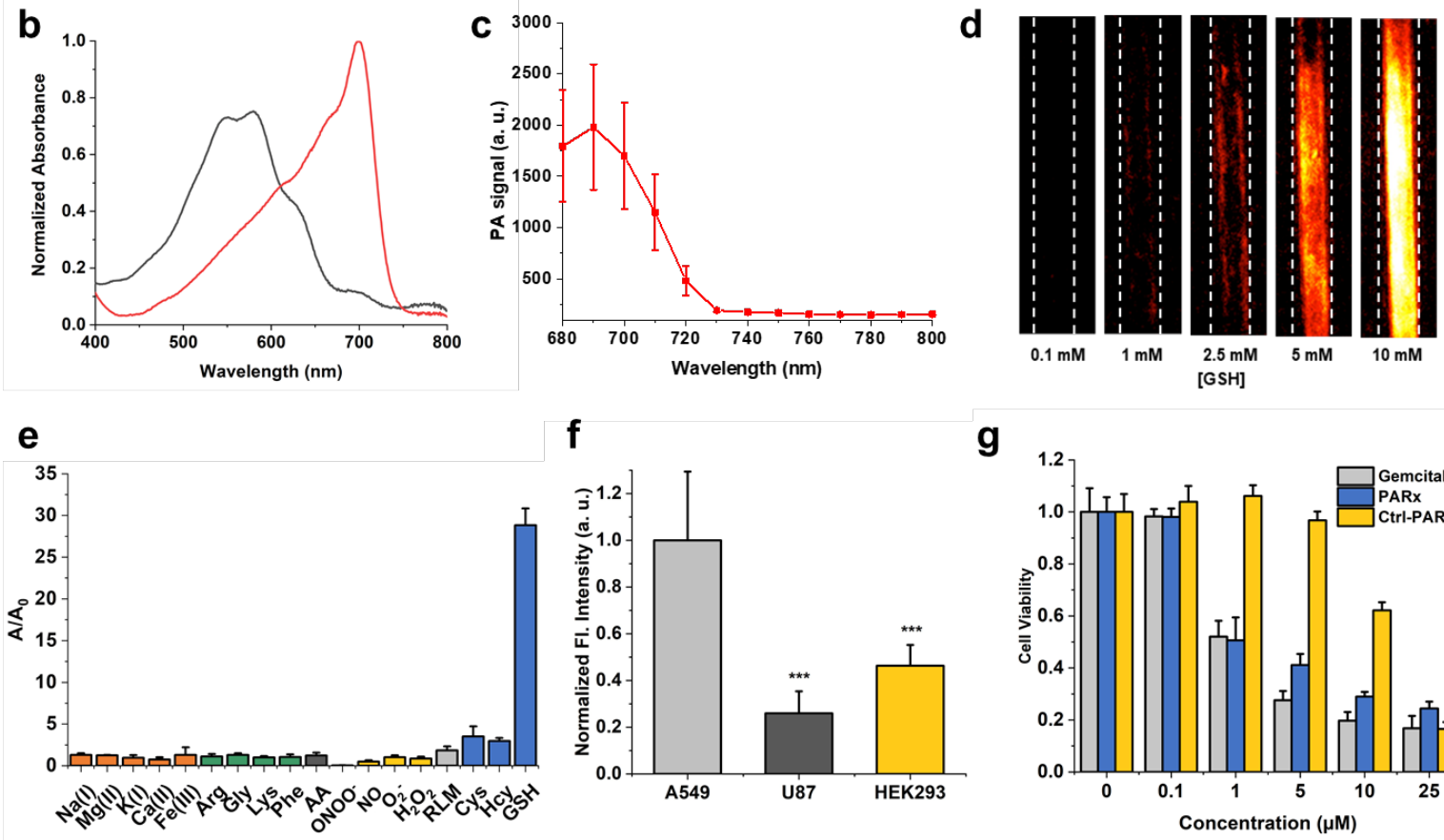

f

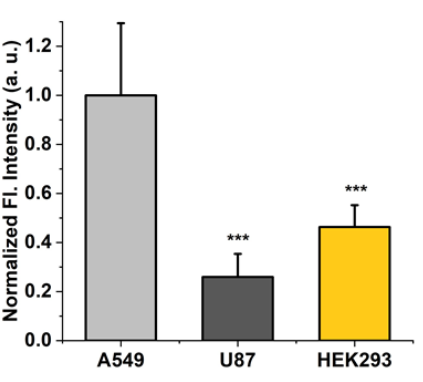

g

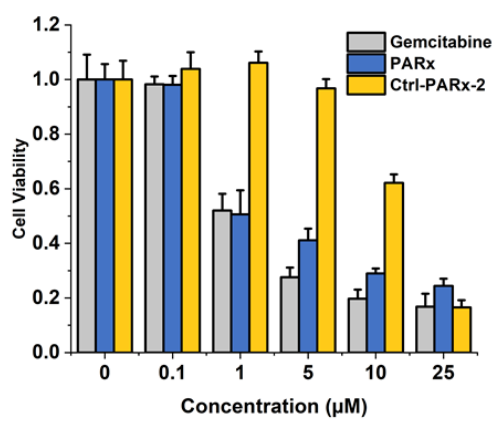

Figure 3. a) Reaction scheme of PARx with GSH to release $\mathrm{HD}-\mathrm{CH}_{2} \mathrm{OH}$ and Gemcitabine. b) Normalized absorbance profile of $5 \mu \mathrm{M}$ PARx before (black line) and after (red line) treatment with $10 \mathrm{mM}$ GSH at 37

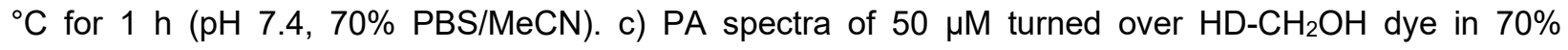
PBS/MeCN. d) PA images of PARx in response to GSH. e) Reactivity of PARx with biologically relevant metals, amino acids, rat liver microsomes, ascorbic acid, RNS, ROS, and thiols after $1 \mathrm{~h}$ incubation at 37 ${ }^{\circ} \mathrm{C}$. f) Normalized fluorescence intensity obtained from cell imaging A549, U87, and HEK293 cells with 5 $\mu \mathrm{M}$ PARx for $1 \mathrm{~h}$ at $37^{\circ} \mathrm{C}$. Error bars $=\mathrm{SD}(n \geq 3)$. g) Cell viability assay using various concentrations of Gemcitabine, PARx, and Ctrl-PARx-2 after $48 \mathrm{~h}$ incubation with A549 cells. Statistical analysis was performed using Student's t-test, ${ }^{* *}: p<0.001,{ }^{* *}: p<0.01,{ }^{*}: p<0.05$. 

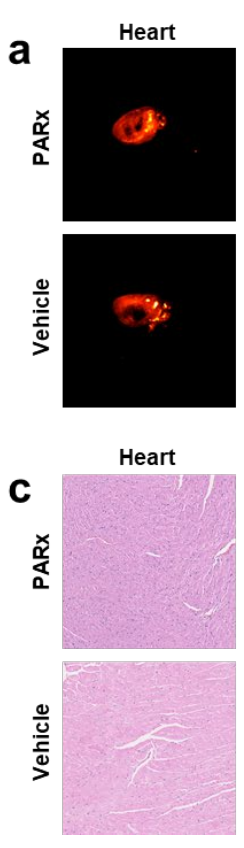

Kidneys
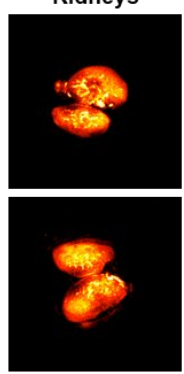

Kidneys
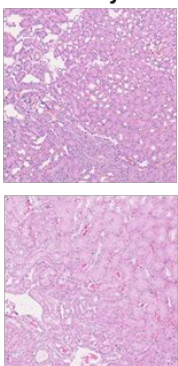

Liver
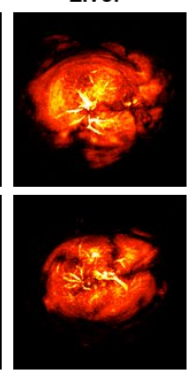

Liver

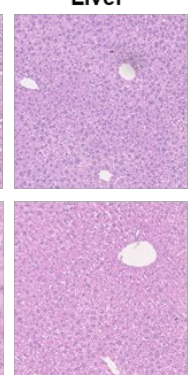

Spleen
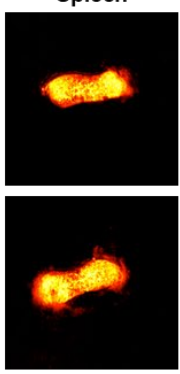

Spleen

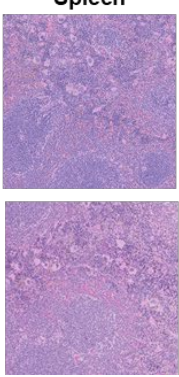

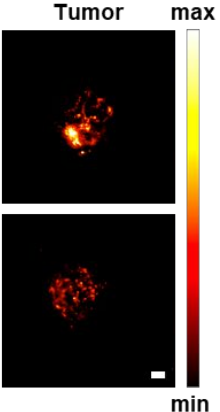

Tumor

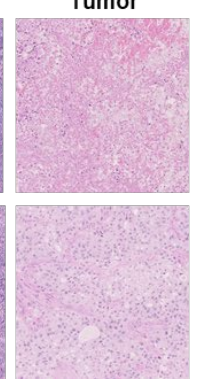

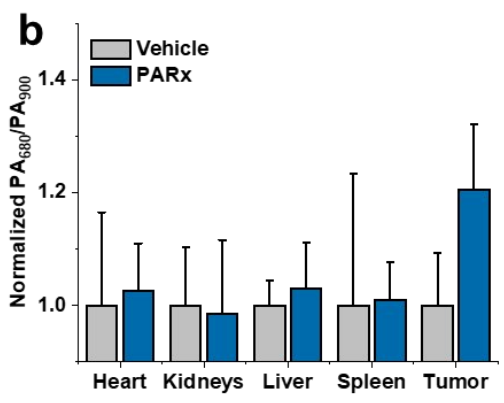

d

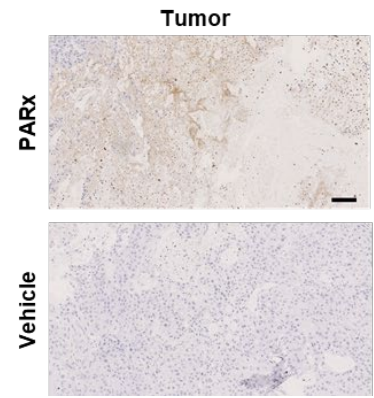

Figure 4. a) Representative ex vivo PA images of heart, kidneys, liver, spleen and tumor after systemic injection of PARx (400 $\mu \mathrm{M}, 10 \%$ DMSO/PBS, retro-orbital injection) or vehicle. Scale bar represents $2 \mathrm{~mm}$. b) Normalized PA signal (relative to a reference probe-independent PA signal) after systemic injection of PARx or vehicle. Error bars = SD $(n=3)$. c) H\&E staining of heart, kidney, liver, spleen, and tumor tissue from PARx-treated and untreated A549 xenografts. d) TUNEL staining of tumor tissue from PARx-treated and untreated A549 xenografts. Brown staining indicates apoptotic cell death. Scale bar represents 100 $\mu \mathrm{m}$. 

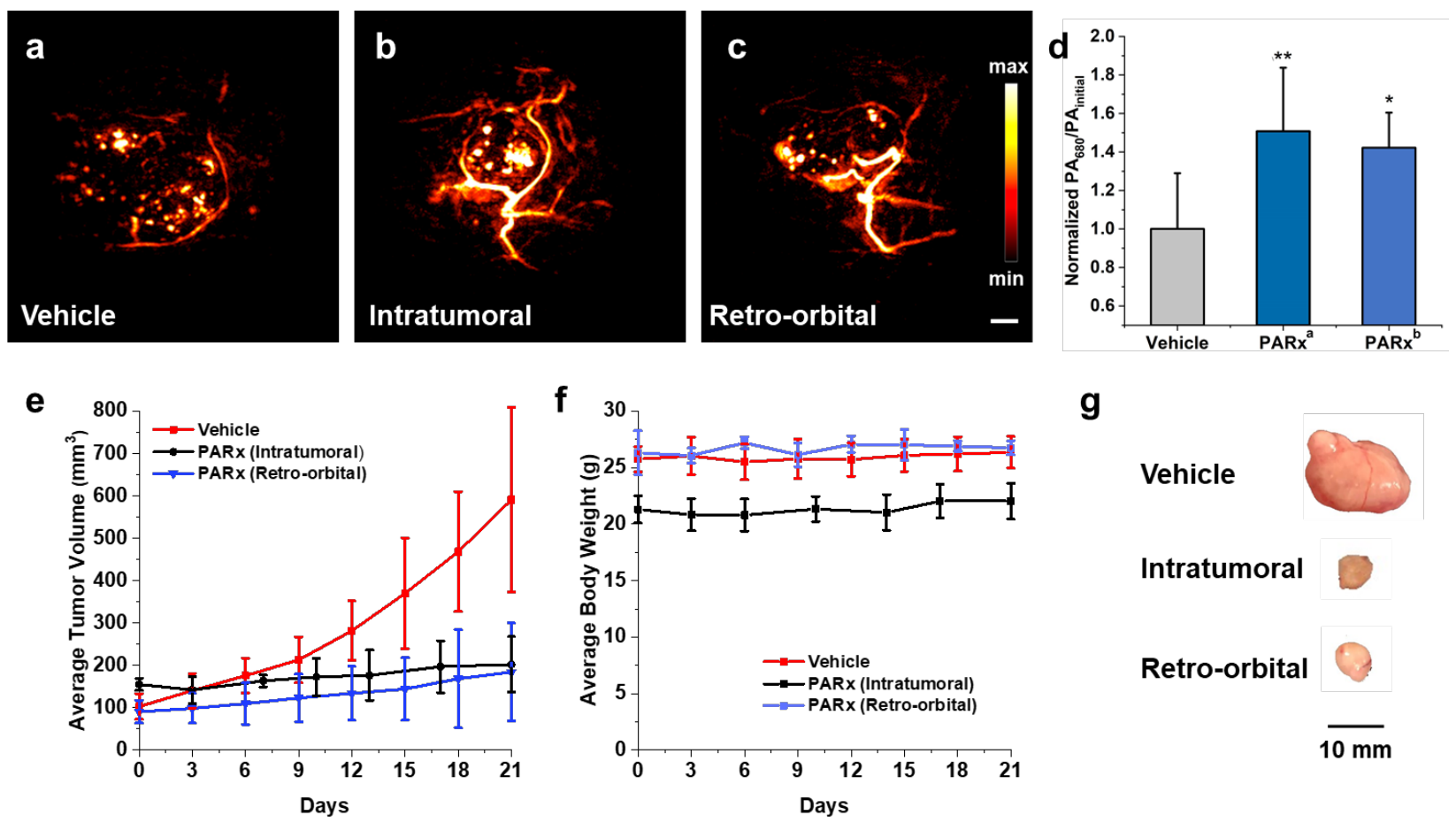

g

Vehicle

\section{Intratumoral}

Retro-orbital

$\overline{10 \mathrm{~mm}}$

Figure 5. PA images of tumors after a) treatment with vehicle (10\%DMSO/PBS), b) intratumoral injection of PARx (100 $\mu \mathrm{M}, 10 \%$ DMSO/PBS), or c) retro-orbital injection of PARx (400 $\mu \mathrm{M}, 10 \%$ DMSO/PBS). Scale bar represents $2 \mathrm{~mm}$. d) Normalized PA signal after treatment with vehicle or PARx. a: intratumoral injection. b: retro-orbital injection. Error bars $=\operatorname{SD}\left(n=3\right.$, tumor volume $\left.<200 \mathrm{~mm}^{3}\right)$. e) Average tumor volume after treatment with vehicle, intratumoral, or retro-orbital injection of PARx over 21 days. f) Average body weight after treatment with vehicle, intratumoral, or retro-orbital injection of PARx over 21 days. g) Representative tumors that were treated with vehicle, intratumoral, or retro-orbital injection of PARx. Statistical analysis was performed using Student's t-test, ${ }^{* *}: p<0.01,{ }^{*}: p<0.05$. 

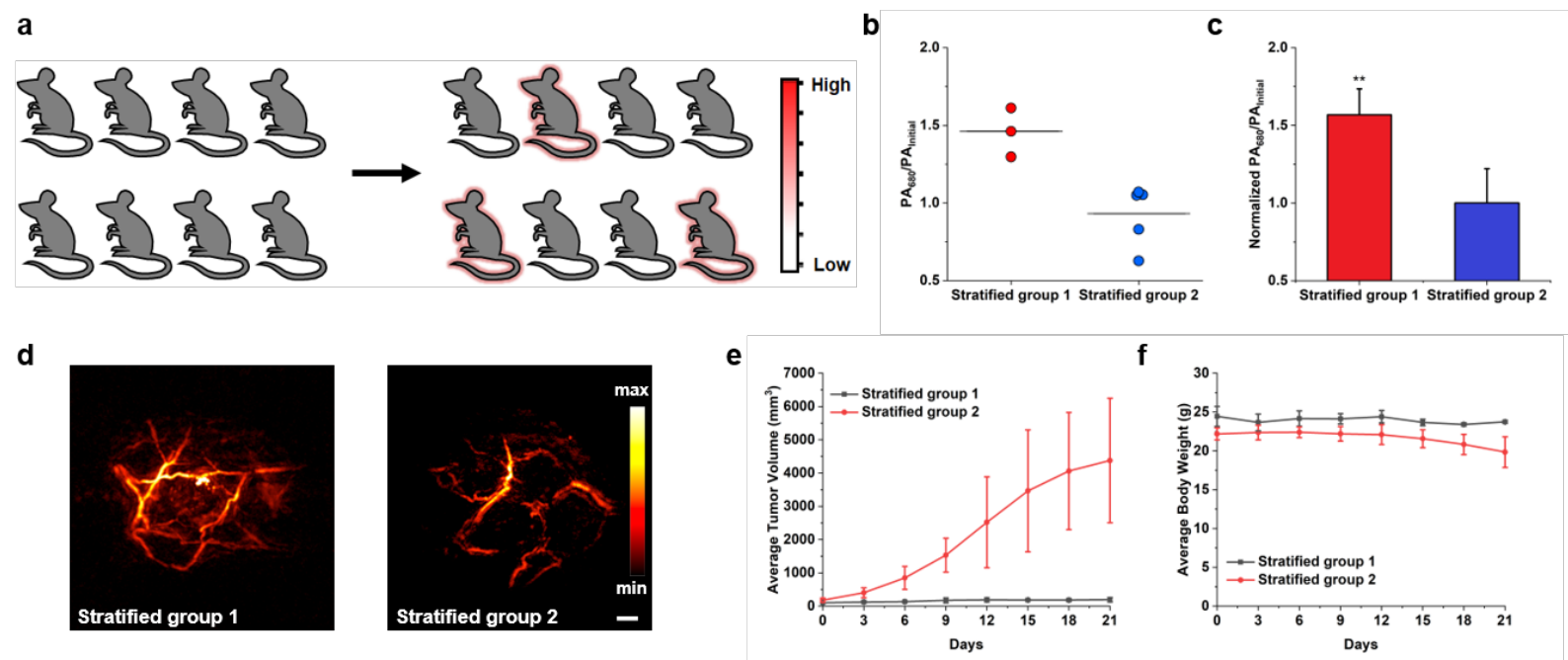

Figure 6. a) Schematic illustrating the stratification of a patient group based on PA signal intensity in a blind study. b) Stratification based on PA fold turn-on after retro-orbital injection of PACDx. c) Normalized PA turn-on of data shown in Figure 6b. d) Representative PA images of stratified groups 1 and 2 after administration of PACDx. Scale bar represents $2 \mathrm{~mm}$. e) Average tumor volume and f) body weights of stratified groups 1 and 2 during a 21-day treatment period with PARx. Errors bars = SD. Statistical analysis was performed using Student's t-test, ${ }^{* *}: p<0.01,{ }^{*}: p<0.05$. 


\section{References}

1 Siegel, R. L., Miller, K. D. \& Jemal, A. Cancer statistics, 2020. CA: A Cancer Journal for Clinicians 70, 7-30, doi:10.3322/caac.21590 (2020).

2 Dracopoli, N. C. \& Boguski, M. S. The Evolution of Oncology Companion Diagnostics from Signal Transduction to Immuno-Oncology. Trends in Pharmacological Sciences 38, 41-54, doi:10.1016/j.tips.2016.09.007 (2017).

3 Agarwal, A., Ressler, D. \& Snyder, G. The current and future state of companion diagnostics. Pharmgenomics Pers Med 8, 99-110, doi:10.2147/PGPM.S49493 (2015).

4 Wang, L. V. \& Hu, S. Photoacoustic Tomography: In Vivo Imaging from Organelles to Organs. Science 335, 1458, doi:10.1126/science.1216210 (2012).

5 Heijblom, M. et al. Photoacoustic image patterns of breast carcinoma and comparisons with Magnetic Resonance Imaging and vascular stained histopathology. Scientific Reports 5, 11778, doi:10.1038/srep11778 (2015).

6 Yang, M. et al. Photoacoustic/ultrasound dual imaging of human thyroid cancers: an initial clinical study. Biomed. Opt. Express 8, 3449-3457, doi:10.1364/BOE.8.003449 (2017).

7 Jo, J. et al. A Functional Study of Human Inflammatory Arthritis Using Photoacoustic Imaging. Scientific Reports 7, 15026, doi:10.1038/s41598-017-15147-5 (2017).

8 Liu, Y., Zhang, L., Li, S., Han, X. \& Yuan, Z. Imaging molecular signatures for clinical detection of scleroderma in the hand by multispectral photoacoustic elastic tomography. Journal of Biophotonics 11, e201700267, doi:10.1002/jbio.201700267 (2018).

9 Reinhardt, C. J. \& Chan, J. Development of Photoacoustic Probes for in Vivo Molecular Imaging. Biochemistry 57, 194-199, doi:10.1021/acs.biochem.7b00888 (2018).

10 Knox, H. J. \& Chan, J. Acoustogenic Probes: A New Frontier in Photoacoustic Imaging. Accounts of Chemical Research 51, 2897-2905, doi:10.1021/acs.accounts.8b00351 (2018).

11 Li, H., Zhang, P., Smaga, L. P., Hoffman, R. A. \& Chan, J. Photoacoustic Probes for Ratiometric Imaging of Copper(II). Journal of the American Chemical Society 137, 15628-15631, doi:10.1021/jacs.5b10504 (2015).

12 Mishra, A., Jiang, Y., Roberts, S., Ntziachristos, V. \& Westmeyer, G. G. Near-Infrared Photoacoustic Imaging Probe Responsive to Calcium. Analytical Chemistry 88, 10785-10789, doi:10.1021/acs.analchem.6b03039 (2016).

13 Roberts, S. et al. Calcium Sensor for Photoacoustic Imaging. Journal of the American Chemical Society 140, 2718-2721, doi:10.1021/jacs.7b03064 (2018).

14 Wang, S. et al. Activatable Small-Molecule Photoacoustic Probes that Cross the Blood-Brain Barrier for Visualization of Copper(II) in Mice with Alzheimer's Disease. Angewandte Chemie International Edition 58, 12415-12419, doi:10.1002/anie.201904047 (2019).

15 Knox, H. J. et al. A bioreducible N-oxide-based probe for photoacoustic imaging of hypoxia. Nature Communications 8, 1794, doi:10.1038/s41467-017-01951-0 (2017). 
16 Knox, H. J., Kim, T. W., Zhu, Z. \& Chan, J. Photophysical Tuning of N-Oxide-Based Probes Enables Ratiometric Photoacoustic Imaging of Tumor Hypoxia. ACS Chemical Biology 13, 1838-1843, doi:10.1021/acschembio.8b00099 (2018).

17 Chen, M. et al. Simultaneous photoacoustic imaging of intravascular and tissue oxygenation. Opt. Lett. 44, 3773-3776, doi:10.1364/OL.44.003773 (2019).

18 Zhou, E. Y., Knox, H. J., Liu, C., Zhao, W. \& Chan, J. A Conformationally Restricted Aza-BODIPY Platform for Stimulus-Responsive Probes with Enhanced Photoacoustic Properties. Journal of the American Chemical Society 141, 17601-17609, doi:10.1021/jacs.9b06694 (2019).

Yin, L. et al. Quantitatively Visualizing Tumor-Related Protease Activity in Vivo Using a Ratiometric Photoacoustic Probe. Journal of the American Chemical Society 141, 3265-3273, doi:10.1021/jacs.8b13628 (2019).

20 Levi, J. et al. Design, Synthesis, and Imaging of an Activatable Photoacoustic Probe. Journal of the American Chemical Society 132, 11264-11269, doi:10.1021/ja104000a (2010).

Reinhardt, C. J., Zhou, E. Y., Jorgensen, M. D., Partipilo, G. \& Chan, J. A Ratiometric Acoustogenic Probe for in Vivo Imaging of Endogenous Nitric Oxide. Journal of the American Chemical Society 140, 1011-1018, doi:10.1021/jacs.7b10783 (2018).

Reinhardt, C. J., Xu, R. \& Chan, J. Nitric oxide imaging in cancer enabled by steric relaxation of a photoacoustic probe platform. Chemical Science 11, 1587-1592, doi:10.1039/C9SC05600A (2020).

Chen, Z. et al. An Optical/Photoacoustic Dual-Modality Probe: Ratiometric in/ex Vivo Imaging for Stimulated H2S Upregulation in Mice. Journal of the American Chemical Society 141, 1797317977, doi:10.1021/jacs.9b09181 (2019).

Forman, H. J., Zhang, H. \& Rinna, A. Glutathione: Overview of its protective roles, measurement, and biosynthesis. Molecular Aspects of Medicine 30, 1-12, doi:https://doi.org/10.1016/j.mam.2008.08.006 (2009).

25 Balendiran, G. K., Dabur, R. \& Fraser, D. The role of glutathione in cancer. Cell Biochemistry and Function 22, 343-352, doi:10.1002/cbf.1149 (2004).

26 Gamcsik, M. P., Kasibhatla, M. S., Teeter, S. D. \& Colvin, O. M. Glutathione levels in human tumors. Biomarkers 17, 671-691, doi:10.3109/1354750X.2012.715672 (2012).

27 Russo, A., DeGraff, W., Friedman, N. \& Mitchell, J. B. Selective Modulation of Glutathione Levels in Human Normal \&lt;em\&gt;versus\&lt;/em\&gt; Tumor Cells and Subsequent Differential Response to Chemotherapy Drugs. Cancer Research 46, 2845 (1986).

Giustarini, D. et al. Glutathione, glutathione disulfide, and S-glutathionylated proteins in cell $\begin{array}{llll}\text { cultures. Free Radical Biology and Medicine 89, 972-981, } & \text {, }\end{array}$ doi:https://doi.org/10.1016/j.freeradbiomed.2015.10.410 (2015).

29 Zhang, J. et al. Synthesis and Characterization of a Series of Highly Fluorogenic Substrates for Glutathione Transferases, a General Strategy. Journal of the American Chemical Society 133, 14109-14119, doi:10.1021/ja205500y (2011).

30 Shibata, A. et al. Fluorogenic probes using 4-substituted-2-nitrobenzenesulfonyl derivatives as caging groups for the analysis of human glutathione transferase catalyzed reactions. Analyst 138, 7326-7330, doi:10.1039/C3AN01339A (2013). 
31 Lee, M. H. et al. Disulfide-Cleavage-Triggered Chemosensors and Their Biological Applications. Chemical Reviews 113, 5071-5109, doi:10.1021/cr300358b (2013).

32 Maeda, H. et al. 2,4-Dinitrobenzenesulfonyl Fluoresceins as Fluorescent Alternatives to Ellman's Reagent in Thiol-Quantification Enzyme Assays. Angewandte Chemie International Edition 44, 2922-2925, doi:10.1002/anie.200500114 (2005).

33 Hansch, C., Leo, A. \& Taft, R. W. A survey of Hammett substituent constants and resonance and field parameters. Chemical Reviews 91, 165-195, doi:10.1021/cr00002a004 (1991).

34 van lersel, M. L. P. S. et al. Inhibition of glutathione S-transferase activity in human melanoma cells by $\alpha, \beta$-unsaturated carbonyl derivatives. Effects of acrolein, cinnamaldehyde, citral, crotonaldehyde, curcumin, ethacrynic acid, and trans-2-hexenal. Chemico-Biological Interactions 102, 117-132, doi:https://doi.org/10.1016/S0009-2797(96)03739-8 (1996).

35 Rahman, I., Kode, A. \& Biswas, S. K. Assay for quantitative determination of glutathione and glutathione disulfide levels using enzymatic recycling method. Nature Protocols 1, 3159-3165, doi:10.1038/nprot.2006.378 (2006).

36 Manegold, C. Gemcitabine (Gemzar®) in non-small cell lung cancer. Expert Review of Anticancer Therapy 4, 345-360, doi:10.1586/14737140.4.3.345 (2004).

37 Hayashi, H., Kurata, T. \& Nakagawa, K. Gemcitabine: Efficacy in the Treatment of Advanced Stage Nonsquamous Non-Small Cell Lung Cancer. Clinical Medicine Insights: Oncology 5, CMO.S6252, doi:10.4137/CMO.S6252 (2011).

38 Toschi, L., Finocchiaro, G., Bartolini, S., Gioia, V. \& Cappuzzo, F. Role of gemcitabine in cancer therapy. Future Oncology 1, 7-17, doi:10.1517/14796694.1.1.7 (2005).

39 He, M., Xuehong, C. \& Yepeng, L. Small Molecular Gemcitabine Prodrugs for Cancer Therapy. Current Medicinal Chemistry 26, 1-20, doi:http://dx.doi.org/10.2174/0929867326666190816230650 (2019).

40 Ullman-Cullere, M. H. \& Foltz, C. J. Body condition scoring: a rapid and accurate method for assessing health status in mice. Lab Anim Sci 49, 319-323 (1999). 